\title{
Fat Grafting for Facial Contouring
}

\author{
Samuel M. Lam, MD, FACS, FISHRS ${ }^{1}$ \\ 1 Private Practice, Dallas, Texas \\ Facial Plast Surg 2019;35:278-285.
}

\begin{abstract}
Address for correspondence Samuel M. Lam, MD, FACS, FISHRS, Private Practice, 6101 Chapel Hill Blvd., Ste.101, Plano, TX 75093 (e-mail: drlam@lamfacialplastics.com).
\end{abstract}

\begin{abstract}
Keywords

- facial fat grafting

- fat transfer

- fat graft

- facial fillers

- injectable fillers

Fat grafting is a safe, effective method for facial rejuvenation if used to restore lost facial volume, but it is not as a method to correct cutaneous deformities and rhytids, or to counteract gravitational effects. The most significant postoperative complication is weight gain that can make a face look overinflated. Accordingly, it is important to select a safe and appropriate candidate. This article outlines who is a suitable prospect for fat grafting and juxtaposes the two methods of facial volume restoration: fat grafting versus injectable fillers. Then a detailed exposition of the operative technique is outlined, including anesthesia, harvesting, processing, and injection. Finally, this article concludes with a description of the postoperative course and the management of potential complications.
\end{abstract}

Facial fat grafting has become a vital component to the armamentarium of many facial plastic surgeons who strive to offer a complete and balanced rejuvenation of the face and neck. Historically, rejuvenative facial work centered entirely on excessive tissue, gravity, and rhytids, with volume loss only being appreciated in the past two decades. However, with the advent of surgical fat grafting and nonsurgical injectable fillers, there has been a rising tide of overfilled faces that have made physicians increasingly wary of volume restoration as a viable method. In addition, many earlier attempts of periorbital fat grafting led to unsightly and difficult-to-correct contour problems that dissuaded many surgeons from performing this procedure. Today, for many facial plastic surgeons, fat grafting represents the surgical method for volume restoration that should be complemented as needed by other techniques such as blepharoplasty, rhytidectomy, and skin resurfacing, depending on the particular nuances of an individual's aging. ${ }^{1-3}$

Along the themes of this issue, many patients who might benefit from lifting or excisional procedures simply will never entertain those options due to fear of undergoing the knife or the belief that noncutting options would be sufficient to make them happy. It is vital that fat grafting and fillers should not be communicated as a method to lift tissues by virtue of filling because it falls far short to accomplish that goal. Patients could be both overfilled and underwhelmed when volume is used to accomplish antigravitational objec- tives. Nevertheless, volume loss arises far earlier than gravitational issues in the majority of patients, and volume restoration can be beneficial for a wider gamut of ages.

This article will strive to cover the subject of facial fat grafting from a surgeon's frame of thinking: preoperative, operative, and postoperative considerations. In preoperative considerations, the focus will be on selecting an appropriate candidate and communicating effectively with the prospective patient the pros, cons, and limitations of fat grafting. In operative considerations, the objective will be to detail in a stepwise fashion how to harvest, process, and inject fat grafting in a safe and reproducible manner. Finally, in postoperative considerations, the discussion will be undertaken on how to manage the standard postoperative course and how to intervene properly when complications arise.

\section{Preoperative Considerations}

It may be wise to begin with who would not be a suitable candidate and why. The greatest risk for an individual seeking fat grafting is postoperative weight gain. ${ }^{4}$ The reason for this risk is that transplanted fat is not a bioinert substance but truly a live graft. Also, the fat that is taken from donor areas recalcitrant to fat loss, for example, the thighs and abdomen, also exhibit the risk of hypertrophy with weight gain. There is a contention that transplanted fat is replaced with mainly collagen/scar tissue, but this assessment is
Issue Theme Anything But the Knife: Nonsurgical Facial Plastic Surgery; Guest Editors: Deborah Watson, MD, FACS, and David B. Hom, MD, FACS
Copyright (c) 2019 by Thieme Medical Publishers, Inc., 333 Seventh Avenue, New York, NY 10001, USA. Tel: +1(212) 584-4662. ISSN 0736-6825. 
inaccurate. There is a tendency of the face to look overfilled and distorted if postoperative weight gain occurs. With subsequent weight loss, individuals return to an appropriate countenance. The question naturally is how much weight gain would be deemed safe and acceptable. As a general rule, percentage of body weight rather than absolute weight change should serve as a guide. For example, someone who weighs approximately 100 pounds may look overinflated if he/she gains only 10 pounds, whereas someone 150 pounds or more may do fine with a 10 -pound weight gain or even more. The surgeon should take a thorough history as to the person's prior weight fluctuations. If the person is trying to lose weight, it is usually preferable to perform the fat transfer about a third of the way into the desired weight loss since many individuals struggle and oftentimes fail to maintain the lower weight profile. Conversely, someone who lacks sufficient donor fat who wants to gain weight for the procedure with the objective to lose the weight postoperatively would not be a good candidate.

Besides a thorough investigation into a patient's weight history, it is important to determine the relative risk of a person's age. If someone is below 40 years of age, fat grafting may not be the ideal choice for facial volumization for a few reasons. First, a younger patient has not experienced metabolic slowdown that could lead to weight gain later in life and thereby imperil the result, as stated previously. Second, many younger patients only exhibit minimal volume loss and might fare better with nonsurgical, office-based fillers. Usually, someone in their thirties who might be a good candidate for fat transfer has been very steady in weight throughout life, has a family history of relatively thinner parental lineage, and exhibits significant volume loss due to premature aging usually caused by excessive sun exposure.

It is important to know that fat grafting undergoes some resorption, the percentage of which is very hard to quantify and is variable in degree. Accordingly, if someone has a small area to be corrected with fat grafting, the concern would be that he/she will be displeased when the transplanted fat partly recedes. Prospective patients should be counseled that fat grafting may be worthwhile if there is more significant global fat loss so that even though there will be partial resorption of transplanted fat, the global improvement will still justify the value of having undergone a fat-transfer procedure. Second, there is a cost associated with undergoing anesthesia along with the need for a more substantive recovery period with fat grafting compared with officebased fillers. Therefore, if someone has more limited fat loss, then he/she should be encouraged to consider having facial fillers instead for all of the reasons outlined previously. The natural challenge that has been raised by patients is that fat is permanent and fillers are temporary, and therefore the patient still prefers fat grafting even if it is a smaller, more limited procedure. It has been observed with hyaluronic acid based fillers that there is an ongoing improvement in results with a few repeated sessions where fillers can last over time to a comparable extent as transplanted fat.

There are several exceptions to the aforementioned rule where more limited fat grafting may be indicated. First, if someone has a significant steatoblepharon where fillers would not easily cover the protuberant lower eyelid fat pocket, then a simultaneous transconjunctival blepharoplasty along with periorbital fat transfer is the preferred method to correct this aesthetic problem. Second, if someone is undergoing other surgical procedures such as a chin augmentation or rhytidectomy, then fat grafting is a very reasonable small addition to the larger procedure. Third, in rare individuals who have had serious repeated infectious issues with fillers, then fat grafting is the only reasonable volume solution for their issues.

Besides some of the inherent risks of fat grafting, it is worth stating when fat grafting may be of limited benefit. Transplanted fat should be placed relatively deeply into the subcutaneous plane (or deeper around the eyes, as will be discussed), and fat is also very soft and pliable as a material. Accordingly, fat is not a good substance to correct nasolabial grooves, to fix acne scars, or to lift superficial cutaneous defects. Furthermore, the postoperative edema following fat grafting in lips can be deforming and protracted with a high resorption rate. Furthermore, as mentioned previously, fat may exhibit the risk of hypertrophy postoperatively due to weight gain. Therefore, it may not be advisable to use fat as a reconstructive tool to fill in asymmetric facial defects, especially in the younger patient and/or in the patient with a highly unstable weight history. ${ }^{5}$ Where fat fails, hyaluronic acid fillers may be used to achieve the desired ends and, as stated, oftentimes can be quite durable after repeated sessions. In fact, it is important for patients who are electing to undergo fat grafting to know that they would most likely benefit from filler treatments toward the end of the first postoperative year for two reasons. First, there will be some absorption of the fat. Second, in the future, the patient will experience further aging even on a yearly basis that would be better treated with officebased fillers. Multiple fat graft sessions are not recommended because additional fat grafting sessions may lead to one of two outcomes: an overfilled face or a desire for still additional fat grafting procedures because there would be once again partial absorption.

For patients who are uncertain whether to pursue fat grafting or fillers, it may be advisable that if they do not undergo fat grafting after a year or so, then they should probably just opt for facial fillers to start looking better sooner. If they say that they want to try fillers first, it is considered less ideal to start with fillers because the patient must have their fillers they paid for dissolved before he/she undergoes fat grafting or wait quite for some time to undergo a fat transfer. As stated, fillers can last months to years, and if a fat transfer is undertaken a few months after fillers, then the fat may appear to dissipate more rapidly because, in fact, the fillers are the part actually fading. Accordingly, fat grafting followed by fillers months to a year later may be preferable, but it is not the other way around. For people who have had a lot of fillers, it could be argued that they simply do not need a fat transfer because they already look exceptional. In short, fillers and fat can achieve comparable results in the right hands. To help patients decide better between the two options, a chart (-Table $\mathbf{1}$ ) that is on the touchscreen 
Table 1 From my web site: comparison chart between fat grafting versus fillers

\begin{tabular}{|c|c|c|}
\hline & Fat grafting & Fillers \\
\hline Type of procedure & Surgical (but no incisions) & Office-based \\
\hline Ideal age & $\begin{array}{l}\text { Over } 35 \text { y old (with enough facial volume loss to } \\
\text { justify the procedure, relatively stable weight, and } \\
\text { healthy to tolerate anesthesia) }\end{array}$ & Any age \\
\hline $\begin{array}{l}\text { Short-term } \\
\text { (initial) cost }\end{array}$ & Higher & Lower \\
\hline Long-term cost & Lower & Higher \\
\hline Recovery & $\begin{array}{l}\text { 7-10 d: you will not look socially acceptable during } \\
\text { the first week but typically have very little dis- } \\
\text { comfort during that time. You should take at least a } \\
\text { week off from work. }\end{array}$ & $\begin{array}{l}\text { Typically, mild swelling for a day or so, but if there is } \\
\text { bruising, it could last in rare cases over a week to } \\
\text { two. Ideally, you should give } 2 \text { wk before major } \\
\text { events, a week before minor events, but many } \\
\text { return to work the same day or the next day if they } \\
\text { are willing to accept some mild swelling and } \\
\text { possibly some minor bruising. }\end{array}$ \\
\hline Benefits & $\begin{array}{l}\text { 1. Can provide the most cost-effective solution for } \\
\text { an individual with moderate-to-significant } \\
\text { volume loss } \\
\text { 2. Permanent solution minus some initial resorp- } \\
\text { tion and also further aging } \\
\text { 3. Can provide improvement in the quality of skin } \\
\text { over time ('stem cell' effect) }\end{array}$ & $\begin{array}{l}\text { 1. Can be tailored to one's budget (small, moder- } \\
\text { ate, or more) } \\
\text { 2. Typically, very little recovery } \\
\text { 3. Can be effective to treat areas in which fat does } \\
\text { not fix well, such as lips and folds } \\
\text { 4. Office-based without the need for anesthesia } \\
\text { 5. Not weight-dependent } \\
\text { 6. Can last many years after a few rounds } \\
\text { 7. Reversible, dissolvable }\end{array}$ \\
\hline Limitations & $\begin{array}{l}\text { 1. More recovery time than fillers (see above) } \\
\text { 2. Some initial resorption of fat } \\
\text { 3. Does not effectively manage smile lines or lips } \\
\text { 4. Fat is dependent on weight, with too much } \\
\text { weight loss the fat can look partially resorbed, or } \\
\text { worse with weight gain the face can look too full }\end{array}$ & $\begin{array}{l}\text { 1. Can be costlier over time when trying to simu- } \\
\text { late a fat transfer } \\
\text { 2. Can take multiple rounds of fillers to achieve } \\
\text { longevity, and therefore multiple smaller rounds } \\
\text { of recovery can exceed the one larger recovery } \\
\text { time of fat transfer } \\
\text { 3. Very low risk (but still possible) of infection since } \\
\text { it is not one's own tissue }\end{array}$ \\
\hline
\end{tabular}

Note: There are two principal ways to restore facial volume: fat grafting and facial fillers. This is oftentimes a confusing decision for many patients. The table lists the pros and cons of each method based on Dr. Lam's experience. Even though this table outlines some of the basic pros and cons of each method, only a consultation with Dr. Lam can help establish with you which method would be better in your case.

consultation program in the author's office and also under both the fat grafting and fillers sections of his web site is used as a simple graphic.

There has also been an increased interest in so-called nanofat injections, which involves highly emulsified fat, used to improve skin texture, scars, and other cutaneous issues. ${ }^{6}$ The author does not have any clinical experience using nanofat and defers this expertise to his colleagues who perform this procedure. He has observed that after many years doing fat transfer, there is a finer texture to the skin under areas in which fat has been transplanted. However, it would be advisable to avoid using hyperbolic marketing terms that emphasize stem cell rejuvenation of transplanted fat.

\section{Operative Considerations}

\section{Anesthesia}

Although fat grafting can be accomplished with the patient fully awake, it may be advisable to perform the procedure with the patient either sedated or under general anesthesia for improved patient comfort. The deeper the sedation, the less anesthetic infiltration is required, and therefore, there can be less ecchymosis related to significant tissue infiltration with local anesthesia. The anesthetic mixture outlined for donor and recipient sites is tailored for some level of patient sedation, whether intravenous or general. With the patient under adequate anesthesia, the physician prepares the donor and recipient sites with povidone-iodine solution. The areas are then sterilely draped.

Then, a mixture of $5 \mathrm{~mL}$ of $1 \%$ lidocaine with 1:100,000 epinephrine together with $15 \mathrm{~mL}$ of plain saline is all that is necessary. Half of the $20-\mathrm{mL}$ mixture is placed with a long spinal needle ( 22 gauge, $5^{\prime \prime}$ ) deeper to the fat plane, and the other half of the anesthetic is distributed more superficially into the immediate subcutaneous plane. For example, if the lower abdomen is harvested, a total of $20 \mathrm{~mL}$ is used for anesthesia in that area, whereas each inner thigh would require $20 \mathrm{~mL}$ per side. If the anterior and lateral thighs are intended for harvesting, then an additional $20 \mathrm{~mL}$ may be used per side as needed. In general, the maximum dose of lidocaine can be safely exceeded with fat harvesting, as the fat cells absorb most of the lidocaine. By using nontumescent techniques for fat harvesting, fat cell integrity is possibly less compromised, which, in turn, may lead to more predictable, long-term outcomes. 
The face is anesthetized with a regional anesthetic block to minimize discomfort so that the direct infiltration of local anesthesia of standard $1 \%$ lidocaine with $1: 100,000$ epinephrine can be undertaken without much pain. The entry sites for the face are then infiltrated with the same $1 \%$ lidocaine with 1:100,000 epinephrine. Additional anesthetic can be distributed with the same infiltration cannula as used for facial fat injection, as needed, to supplement the initial local anesthesia when the patient can tolerate this injection.

\section{Harvesting}

Harvesting of fat is undertaken by hand rather than by wall suction. Many alternative methods for fat harvesting exist and have worked well for their respective surgeons. The entry site is made with a no.16 Nokor needle at a discreet location, for example, the lower aspect of the umbilicus for the lower abdomen and along the inguinal line for thigh harvesting. A bullet-tipped harvesting cannula (Tulip Medical Inc.) is used to harvest the fat from the donor area in question. The cannula is outfitted on a $10-\mathrm{mL}$ Luer-Lok syringe, and a "Johnnie Lok" device (Tulip Medical Inc.) can be used to help hold negative pressure during harvesting so as to alleviate finger strain.

Some basic pearls and pitfalls for proper fat harvesting should be outlined herein to understand safety guidelines that may not be understood by the surgeon unaccustomed to body lipoharvesting or liposuction. It is important that the surgeon's nondominant hand not be used to tent up the skin during harvesting, which, in turn, can lead to contour irregularities. Instead, the nondominant hand can be used with the palm flat on the skin in the area to be harvested to stabilize the fat pad during cannula movement, but that should be the extent of it. More specifically, during harvesting, a novice surgeon tends forcibly to ram the cannula back and forth with the tip of the cannula abutting fascia in the deep plane or skin in the superficial plane that, in turn, will minimize functional fat yield and worsen patient discomfort. Accordingly, the surgeon should be attentive to remain within the center of the fat plane, allowing the cannula to pass across the entire expanse of the area to be harvested but not short or beyond it.

\section{Processing}

Like harvesting, processing techniques vary between physicians who practice fat transfer (-Fig. 1). Unfortunately, each physician is dogmatic about his/her method as the only truly correct way of processing fat. However, straining, centrifugation, washing, and so on have all worked for the physicians who are seasoned in this discipline. The reader is encouraged to learn the method that works by one physician who has extensive experience and not to experiment too much beyond what has been deemed successful given the questionable nature of longevity by many inexperienced physicians who attempt facial fat grafting.

After the fat is collected, the cannula is removed and replaced with a Luer-Lok plug, and the plunger is removed and replaced with a tight-fitting cap, all of which is undertaken sterilely. Syringes prepared in this way are placed into

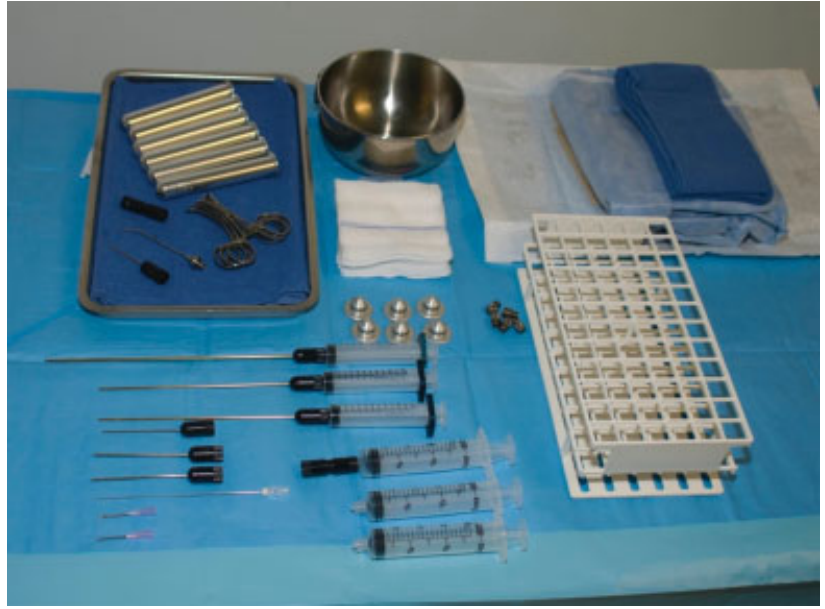

Fig. 1 This figure shows the layout on the sterile surgical table (clockwise from top left): the metallic tray (sleeves for centrifugation, towel clamps, and accessory cannulas), metal bowl into which supranatant/infratant may be discarded, $4 \times 4$ cotton gauze, surgical gown, test-tube rack to be used to place the $10-\mathrm{mL}$ syringes of fat (both pre- and postcentrifugation), $25-\mathrm{mL}$ transfer syringes with one outfitted with a female-to-female transfer adapter, 1.2- and 0.9-mm injection cannulas (bottom left corner), 10-mL syringes with harvesting cannula and Johnnie Lock attached, and the metal caps for centrifugation (center). (Reproduced with permission from Samuel M. Lam, 2019.)

sterile sleeves in a centrifuge and spun for approximately 3 minutes at 3,000 rpm (-Fig. 2). Of course, it is important to ensure that a balanced orientation of syringes is distributed in the centrifuge device. Following centrifugation, the syringes are then sterilely removed. First, the supranatant is poured off of the plunger side, and then the infranatant is drained off from the Luer-Lok side. A sterile test-tube rack is an easy method to keep all of the syringes neatly arranged during this phase of the procedure. Typically, 3 processed $10-$ $\mathrm{mL}$ syringes of collected fat are then placed into a $20-\mathrm{mL}$ syringe by pouring the contents from the open plunger side to open plunger side. The plunger is then inserted into the

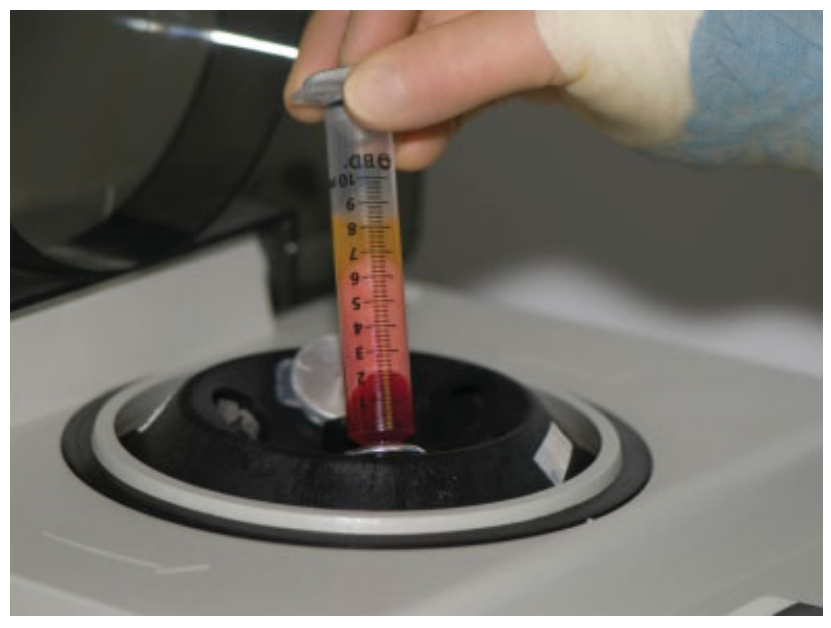

Fig. 2 This photograph shows the 10-mL syringe of fat immediately after centrifugation, removal of the syringe from the sterile sleeve, and the contents with the separated supranatant (above) consisting of lysed fat cells, the central column of fat, and the infranatant (below) consisting of blood and lidocaine. (Reproduced with permission from Samuel M. Lam, 2019.) 
back of the $20-\mathrm{mL}$ syringe taking care not to eject the fat into the air by tilting the Luer-Lok side of the $20-\mathrm{mL}$ syringe upward and sliding the fat back toward the plunger as the plunger is inserted and the air column is advanced out of the syringe. A female-to-female Luer-Lok transfer hub is used to transfer the contents from the $20-\mathrm{mL}$ syringe into individual 1-mL Luer-Lok syringes intended to infiltrate the fat into the face. As the plunger on the $20-\mathrm{mL}$ syringe is depressed, the plunger on the 1-mL syringe is gently glided backward to accommodate the transferred fat all the way until the plunger is actually removed and reinserted back to the 1$\mathrm{mL}$ mark. The infiltration cannula, typically a $1.2-\mathrm{mm}$-wide, straight, and blunt cannula (Tulip Medical Inc.) is outfitted onto the 1-mL Luer-Lok syringe ready for fat injection.

\section{Injection}

With the patient's face properly anesthetized and the fat already processed, fat injection can proceed apace. First, the total fat collected should be measured and quantified as a plan for whether sufficient fat was harvested to complete the job at hand. A status report as the surgeon progresses should be made to ensure that the fat is adequately distributed without running out of transferrable fat. It is typically easier to fill one structure on one side of the face and then the precisely same structure on the other side, for example, lower eyelid orbital rim on the left followed by the same on the right, for four reasons. One, the volumes are immediately recalled so that the same volume is placed per side. Second, immediate recall is still present of how the distribution of fat was made in a particular location, for example, placing more of the $1 \mathrm{~mL}$ of fat toward the lateral brow on the left to be matched on the right side. Third, the facial design can be more readily apparent as the face is slowly built up section by section. Fourth, the fat is less likely to be inadvertently depleted if one side is aggressively filled and then the physician does not have an adequate amount to complete the contralateral side, mandating another harvest for more usable fat.

All entry points are made with a standard 18-gauge needle. An entry point that has worked well for me is the midcheek region to approach the inferior orbital rim, the lateral cheek, the buccal region, and the nasolabial groove. The second entry site is just lateral to the lateral canthus, which allows access to the upper eyelid/brow, temple, and cheek. The third entry site just behind the prejowl sulcus permits augmentation of the prejowl region and the anterior chin.

\section{Inferior Orbital Rim}

This is technically the most demanding area to work on, as too much placed too quickly in the wrong tissue plane can lead to contour problems that are not easy to manage. From the midcheek entry point, the cannula is guided upward toward the orbital rim. The nondominant index finger is used to protect the globe and also to guide placement in the right plane and the right location. The cannula tip should feel like it is releasing some fibrous adhesions so that the tip resides in the immediate supraperiosteal plane. Again, no more than hundredth of a milliliter per pass of the cannula should be used to inject the fat to permit only very small aliquots at any one time. In general, a total of no more than $3 \mathrm{~mL}$ should be placed per side with perhaps another $0.5 \mathrm{~mL}$ targeted specifically in the lateral canthal hollow, which is also more easily approached from a separate lateral entry site. The nondominant index finger can feel that the placement of the fat is just running back and forth across the bony orbital rim by about a millimeter on each side and no farther (-Fig. 3). Additional $1 \mathrm{~mL}$ of fat can be placed into the nasojugal groove or triangular soft tissue defect just inferior to the medial aspect of the inferior orbital rim.

\section{Superior Orbital Rim and Brow}

Rather than looking strictly at dermatochalasis of the upper eyelid, there is oftentimes a flattening of the brow and at times a visible depression of the skin along the superior aspect of the orbital rim. This depression tends to be a focus of concern that can lead to remarkable aesthetic improvement. Approaching the soft tissue loss of the upper eyelid and infrabrow region, the physician can more rapidly place fat across this defect from a lateral entry site at the lateral canthus. On average 1 to $2 \mathrm{~mL}$ works well to augment this region effectively. Some particular regions of the upper eyelid and infrabrow that can be effectively targeted include the medial notching from either aging or surgical fat removal, known as the A-frame deformity, and/or more laterally along the brow that could typically be thought of as brow ptosis

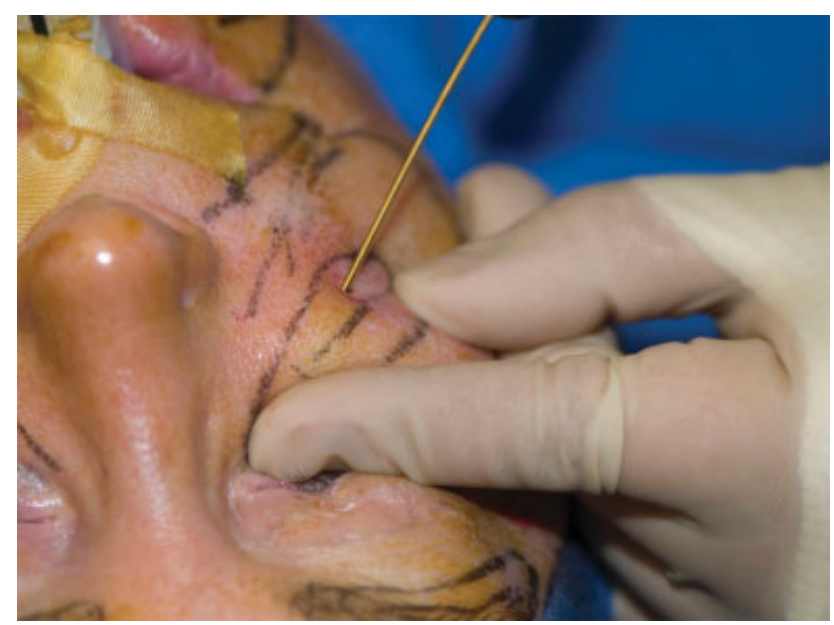

Fig. 3 This is technically the most demanding area to work on, as too much placed too quickly in the wrong tissue plane can lead to contour problems that are not easy to manage. From the midcheek entry point, the cannula is guided upward toward the orbital rim. The nondominant index finger is used to protect the globe and also to guide placement in the right plane and the right location. The cannula tip should feel like it is releasing some fibrous adhesions so that the tip resides in the immediate supraperiosteal plane. Again, no more than a hundredth of a milliliter per pass of the cannula should be used to inject the fat to permit only very small aliquots at any one time. In general, a total of no more than $3 \mathrm{~mL}$ should be placed per side with perhaps another $0.5 \mathrm{~mL}$ targeted specifically in the lateral canthal hollow, which is also more easily approached from a separate lateral entry site. The nondominant index finger can feel that the placement of the fat is just running back and forth across the bony orbital rim by about a millimeter on each side and no farther. (Reproduced with permission from Samuel M. Lam, 2019.) 


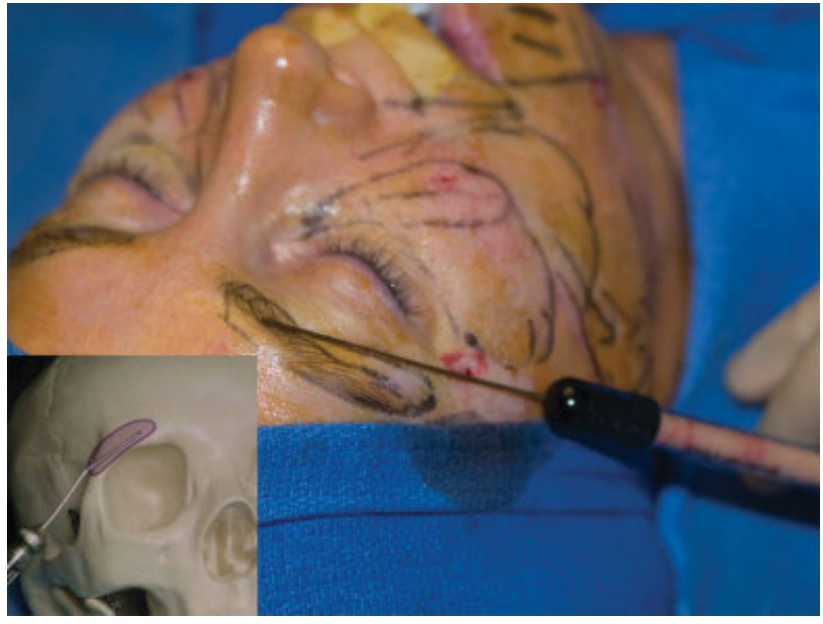

Fig. 4 Rather than looking strictly at dermatochalasis of the upper eyelid, there is oftentimes a flattening of the brow and at times a visible depression of the skin along the superior aspect of the orbital rim. This depression tends to be a focus of concern that can lead to remarkable aesthetic improvement. Approaching the soft tissue loss of the upper eyelid and infrabrow region, the physician can more rapidly place fat across this defect from a lateral entry site at the lateral canthus. On average, 1 to $2 \mathrm{~mL}$ works well to augment this region effectively. Some particular regions of the upper eyelid and infrabrow that can be effectively targeted include the medial notching from either aging or surgical fat removal, known as the A-frame deformity, and/or more laterally along the brow that could typically be thought of as brow ptosis when it could be simply brow deflation. The plane of infiltration is along that of least resistance, typically in the subcutaneous dimension. (Reproduced with permission from Samuel M. Lam, 2019.)

when it could be simply brow deflation. The plane of infiltration is along that of least resistance, typically in the subcutaneous dimension (-Fig. 4).

\section{Temple}

The temple is a relatively advanced area to fill and perhaps should be approached with ongoing sophistication in technique and acquired experience. Just like the hollow inferior orbital rim, only very small aliquots of a hundredth of a milliliter should be passed in the subcutaneous plane to avoid a likely contour problem. Using only 1 to $2 \mathrm{~mL}$ is adequate in most cases to fill each temple. However, experience will dictate the quantity that would be safe and effective in a particular surgeon's hands.

\section{Anterior Cheek}

The anterior cheek should only be filled in rare cases. Fat retention is simply too high in this area, as it can lead to an overfilled appearance, and even 1 to $2 \mathrm{~mL}$ may appear too full in many cases.

\section{Lateral Cheek}

The lateral cheek is the area in continuity with the anterior cheek but lies more laterally across the exposed malar bony prominence. The lateral cheek can be approached easily from the midcheek entry site while aiming the cannula superolaterally. Typically, 1 to $2 \mathrm{~mL}$ is adequate to build the lateral cheek dimension (-Fig. 5).

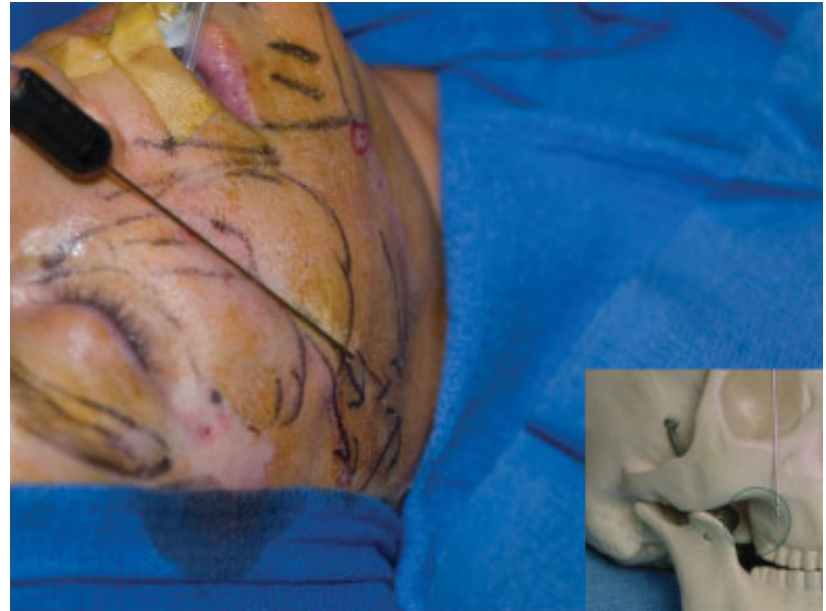

Fig. 5 This photograph shows the placement of fat into the lateral cheek (superior to where the cannula resides in the photograph) and the area directly below the zygomatic arch (where the cannula tip is shown) and contiguous with the lateral cheek fill above. This outer area of filling extending from over the zygomatic arch inferiorly to the arch can be a critical area to fill to create a more attractive and youthful facial shape, the classic oval-shaped face of youth. (Reproduced with permission from Samuel M. Lam, 2019.)

\section{Buccal Area}

In the slightly overweight patient, the buccal area is typically avoided because it can make the individual appear to be even more corpulent. The buccal area can be easily approached from the midcheek entry site aiming inferolaterally in a plane of least resistance in the subcutaneous dimension. Typically, 1 to $4 \mathrm{~mL}$ can be used depending on the degree of hollowness. However, the central buccal area can also contribute to an overfilled appearance when the patient smiles therefore; it is recommended to inject the fat far more laterally under the zygomatic arch to be blended upward with the zygomatic prominence (-Fig. 5).

\section{Prejowl Sulcus}

The area immediately anterior to the jowl reflects a bony depression, which, if filled, can blend the appearance of the jowl better and make the lower face more youthful. In the individual with mild jowling, the prejowl fill with fat can be sufficient to create a less obvious jowl appearance. For the individual with substantial jowling, the result of a lower rhytidectomy can be enhanced by combining the procedure with a fat transfer to the prejowl sulcus. Typically, 2 to $4 \mathrm{~mL}$ can be used along a plane of least resistance to fill in this area with rapid, larger boluses of fat on the order of $1 / 10$ th of a milliliter per pass like in the buccal and cheek areas (-Fig. 6).

\section{Postoperative Considerations}

\section{Postoperative Care}

Since there are no sutures or bandages used, there is very little afterwork required in terms of wound care and so on. However, ice in the face can reduce postoperative edema and thus can reduce excessive valsalva/straining, bending over, or heavy salt diets. There is truly no prohibitive exercise, but care should be taken to not engage in overzealous activity that would 


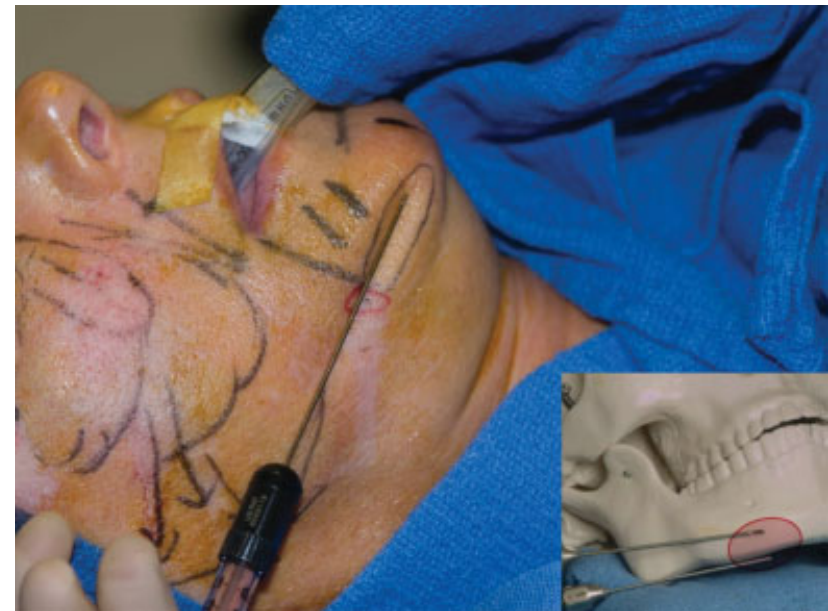

Fig. 6 The area immediately anterior to the jowl reflects a bony depression, which, if filled, can blend the appearance of the jowl better and make the lower face more youthful. In the individual with mild jowling, the prejowl fill with fat can be sufficient to create a less obvious jowl appearance. For the individual with substantial jowling, the result of a lower rhytidectomy can be significantly improved by combining the procedure with a fat transfer to the prejowl sulcus. The physician should imagine the prejowl expanse as a confluence of three cylinders that needs filling: one along the anterior margin of the mandible, a second in the inferior aspect of the mandible (where the jowl is hanging), and a third as a transition between the two areas. Typically, 2 to $4 \mathrm{~mL}$ can be used along a plane of least resistance to fill in this area with rapid, larger boluses of fat on the order of $1 / 10$ th of a milliliter per pass like in the buccal and cheek areas. (Reproduced with permission from Samuel M. Lam, 2019.)

predispose toward prolonged edema. Patients should know that their face will look too swollen typically for the first 1 to 2 weeks and may take as long as 3 to 4 weeks to begin liking the result. As a reminder to the patient, there is a decline in the aesthetic result that can manifest 2 to 3 months following the procedure, but that should gradually improve over the first 6 to 18 months postoperatively, as the graft begins to take hold and attain blood supply. Patients should be evaluated with sequential photographs to show them these changes and should be patiently reminded about the slow process to attain optimal results.

\section{Management of Complications}

Hopefully, this article will serve to provide a basic framework of knowledge to help the beginning surgeon to circumvent easily avoidable mistakes. ${ }^{7}$ Taking care to inject slowly sensitive areas such as the lower eyelid in the prescribed manner can virtually eliminate risks of contour deformities. Conservative volumes of fat transfer can lead to safer outcomes, especially if the physician avoids the temptation for too early a touch-up before the fat grafting result has even begun to manifest. Choosing patients wisely who do not have significant weight fluctuations can be one of the most important ways to preclude a problem that can arise with significant postoperative weight gain following a fat transfer.

Nevertheless, complications can and do occur despite the work of the most technically gifted and precise surgeon. A firm, fibrotic mass that at times can be palpable in the lower eyelid region is because of the placement of the fat in the wrong plane and can typically be corrected with injectable 5fluorouracil or with diluted triamcinolone acetonide. For a visible, rounder lump, the lump should just be excised along a discreet line, for example, the tear trough. If the patient is overfilled, it is very difficult to microliposuction the face of all the excessive fat, but some improvement can be attained. Therefore, it is very important to be conservative and add more fat, which is easy, as needed in the future.

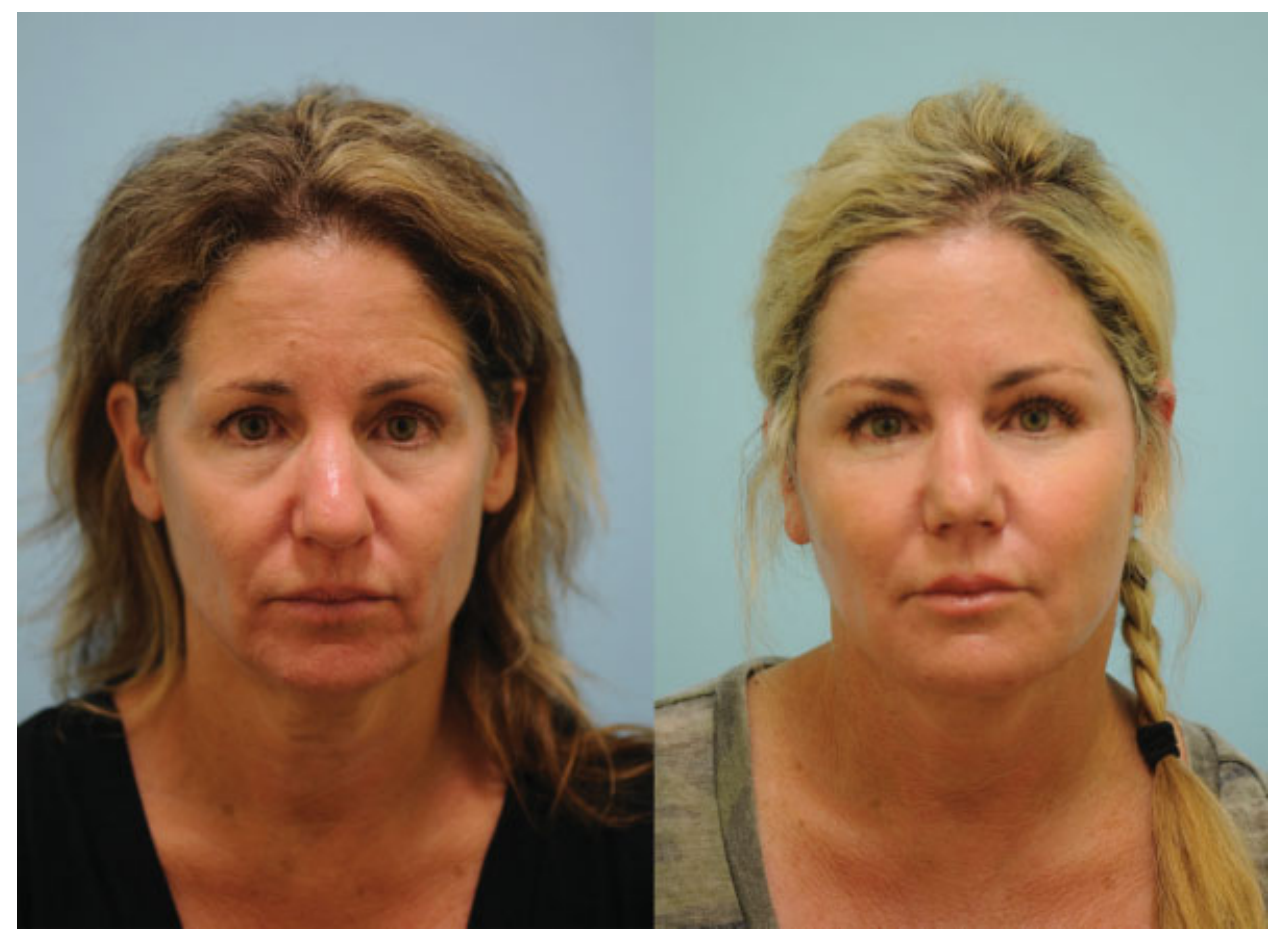

Fig. 7 This 53-year-old woman underwent full-facial fat grafting, rhinoplasty, rhytidectomy, full-facial erbium laser resurfacing, and botulinum toxin treatments to achieve a global rejuvenation. (Reproduced with permission from Samuel M. Lam, 2019.) 

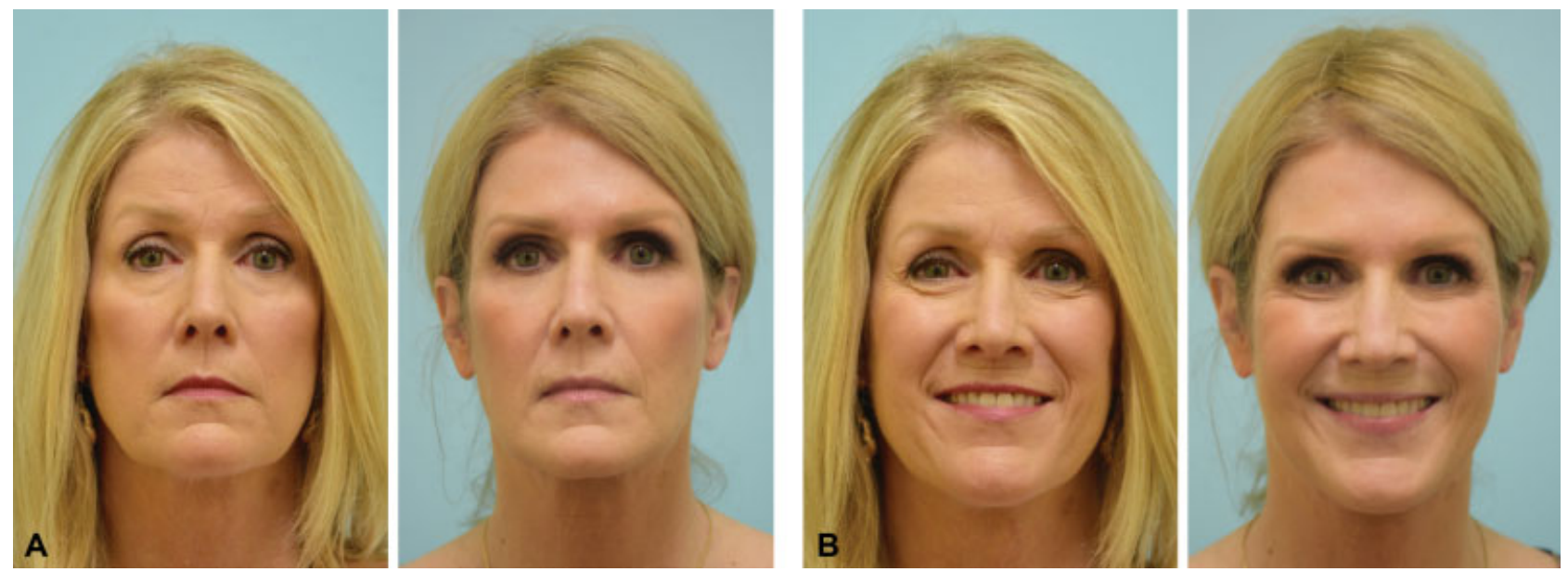

Fig. 8 (A) This 55-year-old woman underwent full-facial fat grafting and is shown before and after with notable softening and rejuvenation of her facial features. (B) She is shown that even while smiling, the cheeks do not look overinflated because no fat was placed into the central and anterior cheek to avoid this type of complication. (Reproduced with permission from Samuel M. Lam, 2019.)

\section{Conclusion}

Fat grafting can be a safe and wonderful intervention for the aging face whether by itself or as a complement to traditional lifting procedures (-Figs. 7, 8). Understanding the way the face ages through volume loss is a prerequisite to begin one's journey toward performing fat transfer for facial rejuvenation. The obsolete idea that fat transfer is dangerous or futile has been replaced with the current protocol that can lead to safety and longevity when the surgeon exercises proper technique, caution, and artistic judgment over time.

\section{Conflict of Interest}

The author has no conflict of interest to disclose.

Acknowledgments

None

\section{References}

1 Lam SM, Glasgold MJ, Glasgold RA. Complementary Fat Grafting. Philadelphia, PA: Lippincott Williams \& Wilkins; 2006

2 Glasgold MJ, Glasgold RA, Lam SM. Volume restoration and facial aesthetics. Facial Plast Surg Clin North Am 2008;16(04):435-442, vi

3 Glasgold RA, Lam SM, Glasgold MJ. Facial fat grafting: the new paradigm. Arch Facial Plast Surg 2008;10(06):417-418

4 Lambros V. Fat grafting: a growing problem? Plast Reconstr Surg 2018;141(02):527-528

5 Krastev TK, Beugels J, Hommes J, Piatkowski A, Mathijssen I, van der Hulst R. Efficacy and safety of autologous fat transfer in facial reconstructive surgery. A systematic review and meta-analysis. JAMA Facial Plast Surg 2018;20(05):351-360

$6 \mathrm{GuZ}$, LiY, Li H. Use of condensed nanofat combined with fat grafts to treat atrophic scars. JAMA Facial Plast Surg 2018;20(02):128-135

7 Lam SM, Glasgold RA, Glasgold MJ. Limitations, complications, and long-term sequelae of fat transfer. Facial Plast Surg Clin North Am 2008;16(04):391-399, v 